\title{
El afecto en la relación docente-estudiante
}

\section{Affection in the Teacher-Student Relationship}

\section{O afeto no relacionamento professor-aluno}

Daissy Andrea Burbano-Fajardo, Psi. *

Sonia Mireya Betancourth-Zambrano, Psi., Msc., PhD. **

\section{Resumen}

Introducción: La relación docente-estudiante es fundamental para los procesos de aprendizaje y desarrollo integral de los estudiantes en el aula. Se dan dos tipos de relaciones: didáctica y socioafectiva. En este sentido, se reconoce que el desarrollo del vínculo afectivo repercute en el desarrollo humano, intelectual, académico, social y religioso. Objetivo: Determinar la relación socioafectiva docenteestudiantes de preescolar y primero de primaria a través de los comportamientos del docente. Metodología: Investigación cuantitativa, de tipo descriptivo exploratorio. La muestra fue por conveniencia conformada por 14 docentes, 9 de preescolar y 5 de primero primaria de dos instituciones de la ciudad de Pasto, Colombia. El instrumento que se utilizó fue el Classroom Assessment Scoring System de Pianta el cual valora tres dominios: el apoyo emocional, organizacional y cognitivo, que contienen 9 dimensiones y con el cual se analizó tres filmaciones de clases por docente, grabadas al inicio, mediados y final del año. El equipo investigador realizó el análisis de los videos simultáneamente, para establecer un acuerdo inter jueces. Se calificó de uno a siete, siendo uno el puntaje más bajo y siete el puntaje mayor, evaluando la relación de los docentes en cada dimensión. Resultados: La relación docente-estudiante planteada por Pianta se analizó teniendo en cuenta el criterio de esfericidad de Mauchly. Es así como se observa que en ocho de las nueve dimensiones no existen diferencias significativas a lo largo del tiempo, por lo tanto las docentes mantienen la misma forma de relacionarse durante todo el año escolar en los aspectos afectivo y de aprendizaje a diferencia de la dimensión del lenguaje donde se observa que si existen diferencias significativas. Conclusiones: Para lograr el progreso positivo en la relación es indispensable involucrar el componente del afecto y la comunicación para conseguir la comprensión mutua, la percepción interpersonal y la empatía, teniendo siempre claro que el afecto mantiene una relación indisociable con la cognición. [Burbano-Fajardo DA, Betancourth-Zambrano SM. MedUNAB 2017-2018; 20(3): 310-318].

Palabras claves: Afecto; Docentes; Cognición; Estudiantes; Educación.

\footnotetext{
* Psicóloga, Universidad de Nariño, Pasto, Nariño, Colombia.

** Psicóloga, magíster en Educación con Énfasis en Docencia Universitaria, doctorado en Psicología Escolar y del Desarrollo. Docente Universidad de Nariño,
} Pasto, Nariño, Colombia.

Correspondencia: Daissy Andrea Burbano-Fajardo.953 Highland Terrace NE, Atlanta, GE. Teléfono: +1 (404) 5427048. E-mail: dabf.andrea@gmail.com. 


\section{Abstract}

Introduction: The teacher-student relationship is vital for the processes of learning and comprehensive development of students within the classroom. There are two types of relationships: didactic and socio-affective. In this sense, the development of the affective bond affects the human, intellectual, academic, social and religious development is recognized. Objective: To determine the teacher-child, from pre-school and first grade (elementary), socio affective relationship through teacher behavior patterns. Methodology: This is a descriptive and exploratory quantitative research. The sample was conformed by 14 teachers, 9 preschoolers, and 5 first graders from two educational institutions in the city of Pasto, Colombia. The instrument that was used was the Classroom Assessment Scoring System developed by Pianta, which evaluates three domains: emotional support, classroom organization and instructional support. These domains have also 9 dimensions. With this instrument, three recorded classes, per teacher, were analyzed. These recordings were filmed at the beginning, in the middle and at the end of the school year. The research team conducted the analysis of the videos simultaneously in order to establish an inter-judge agreement. The score was set from 1 to 7 , being 1 the lowest and 7 the highest score. The purpose of this was to evaluate the teachers' relationship in each dimension. Results: The teacher-student relationship proposed by Pianta was analyzed taking into account Mauchly's sphericity test criterion. It is observed that in eight of the nine dimensions there are no significant differences over time; therefore, teachers keep the same way of being in contact with children throughout the school year in the affective and learning aspects; as opposed to the dimension of language, in which it is observed that there are significant differences indeed. Conclusions: To achieve positive progress in the relationship is essential to involve the component of affection and communication in order to accomplish a mutual understanding, an interpersonal perception and empathy; this can be achieved by having in mind that the affection maintains an unbreakable relationship with the cognitive area. [Burbano-Fajardo DA, BetancourthZambrano SM. Affection in the Teacher-Student Relationship. MedUNAB 2017-2018; 20(3): 310-318].

Keywords: Affect; Faculty; Cognition; Students; Education.

\section{Introducción}

Educar a los niños y niñas es una de las preocupaciones más constantes no solo en una ciudad o país sino alrededor del mundo así, la formación integral del individuo es y será el objetivo principal de cualquier proceso de aprendizaje (1). De esta manera, los docentes de primaria y preescolar reciben una formación universitaria desde hace aproximadamente 40 años (2), en donde aprenden el reconocimiento de la afectividad en el aula de clase (3). Por esta razón, educar en la afectividad no es un tema menor, debido a la gran repercusión que tiene el desarrollo del vínculo afectivo en el desarrollo humano, intelectual, académico, social y religioso (1).

\section{Resumo}

Introdução: a relação professor-aluno é fundamental para os processos de aprendizagem e desenvolvimento integral dos alunos na sala de aula. Existem dois tipos de relacionamentos: didáticos e socio-afetivos. Neste sentido, reconhece-se que o desenvolvimento do vínculo afetivo afeta o desenvolvimento humano, intelectual, acadêmico, social e religioso. Objetivo: Determinar a relação socioafetiva professora-estudante do pré-escolar e do primeiro grau, através do comportamento do professor. Metodologia: pesquisa quantitativa, tipo exploratório descritivo. A amostra foi por conveniência, composta de 14 professores, 9 de pré-escolar e 5 de primeiro grau, de duas instituições da cidade de Pasto, na Colômbia. O instrumento utilizado foi o Sistema de Pontuação de avaliação da sala de aula de Pianta que avalia três áreas: suporte emocional, organizacional e cognitivo, que contém 9 dimensões e com as quais foram analisadas três fitas de video por professor, registradas no início, no meio e no final do ano. A equipe de pesquisa realizou a análise dos vídeos simultaneamente, para estabelecer um acordo entre os juízes. A nota foi de um a sete, sendo um o de menor pontuação e sete o de maior resultado, avaliando a relação dos professores em cada dimensão. Resultados: a relação professora-aluno, proposta por Pianta, foi analisada levando em consideração o critério de esfericidade de Mauchly. É assim que se observa que em oito das nove dimensões não há diferenças significativas ao longo do tempo, portanto, os professores mantêm a mesma maneira de se relacionar ao longo do ano letivo nos aspectos afetivos e de aprendizado em oposição à dimensão da linguagem; na qual se observa que si existem diferenças significativas. Conclusões: Para alcançar um progresso positivo no relacionamento, é essencial envolver o componente de carinho e comunicação para alcançar o entendimento mútuo, a percepção interpessoal e a empatia, sendo muito claro que o carinho mantém uma relação inseparável da cognição. [Burbano-Fajardo DA, Betancourth-Zambrano SM. O afeto no relacionamento professor-aluno. MedUNAB 2017-2018; 20(3): 310-318].

Palavras-chave: Afeto; Docentes; Cognição; Estudantes; Educação.

De esta manera, se retoma la teoría socio constructivista inspirada por Vygotsky (4), quien además de centrarse en que el desarrollo es de naturaleza histórico cultural, manifiesta que la cognición se basa en el afecto. De hecho, el aprendizaje que se inscribe en la trayectoria de desarrollo de los niños es realizado en colaboración con un contexto intersubjetivo (4). Por lo tanto, los seres humanos no pueden ser considerados independientemente de su entorno (5).

La relación entre afectividad y cognición es un fenómeno reconocido por varios autores $(6,7,8)$, y desde la perspectiva de Vygotsky es impensable considerar uno sin el otro "el pensamiento mismo no nace de otro pensamiento, sino de la esfera motivadora de la conciencia, que incluye nuestros impulsos y nuestras necesidades, nuestros intereses y 
nuestros móviles, nuestros afectos y nuestras emociones" (9). Adicionalmente, menciona que uno de los principales defectos de la psicología tradicional es que se separa el afecto de la inteligencia, aspectos que deben estar unidos y que se fortalecen el uno con el otro (10).

Es así como la teoría del apego parece especialmente adecuada para tratar de entender cómo se establece la relación entre el niño y el adulto que lo cuida (11). Aunque esta teoría trata de la génesis de la construcción de las relaciones y, como tal, se centra en la relación que se establece entre el niño y sus padres, da cuenta de cómo se desarrolla la relación entre el niño y su docente, en tanto que es la que garantiza la seguridad del niño en la clase, así como la relación padres-hijos tiene por objeto garantizar la seguridad del niño en su medio familiar. Aunque son diferentes, estas relaciones tienen en común la noción de seguridad. Una madre está atenta a las necesidades de su niño y lo ayuda a desarrollar confianza en sí mismo y en los otros, la relación de apego positivo ayuda al niño a desarrollar su capacidad de explorar el mundo que le rodea. De la misma manera, un docente sensible a las necesidades de sus estudiantes y capaz de responder de manera apropiada, les ayudará a desarrollar su capacidad de explorar el mundo (del conocimiento), y por lo tanto alcanzar su autonomía. Esta analogía se aplica especialmente a los docentes de preescolar y primer año de primaria, ya que son ellos los que proporcionan la transición entre la educación informal y la educación formal (11); además, son quienes reforzarán el vínculo emocional que se establece entre aprendices y maestros.

Desde esta perspectiva, la relación docente-estudiante aparece cada vez más como un elemento de extrema importancia en esencial para el éxito académico de los estudiantes (12). Se ha observado, por ejemplo, que en repetidas ocasiones la calidad de la relación con el docente de preescolar repercute sobre los aprendizajes escolares de los estudiantes, no solamente en preescolar sino hasta el fin de la primaria $(13,14)$ y la educación secundaria. Los adolescentes graduados han tenido un proceso de aprendizaje determinado por el preescolar, lo que hace que el trabajo del docente en las primeras etapas de enseñanza-aprendizaje esté dirigido a estimular el desarrollo cognitivo, emocional, del lenguaje, físico, social, moral y sexual de los niños (15).

Vale mencionar que la relación docente-estudiante, ejerce un efecto particularmente positivo en el rendimiento académico de los estudiantes con dificultades de aprendizaje y adaptación social (16). Se ha observado que la calidad de las relaciones con los docentes de primer año actúa como variable moderadora de la calidad de relación de apego de los niños más pequeños (17). Estos resultados apoyan la idea de que esta relación juega un papel clave en el futuro académico de los estudiantes.

La calidad de la relación docente-estudiante se ha estudiado desde distintas perspectivas; sin embargo, para esta investigación se aborda el tema desde dos grandes campos: la relación didáctica y la relación socioafectiva.

Bajo el ángulo socio afectivo, un gran número de autores han señalado la importancia de la sensibilidad del docente tanto en el plano afectivo (18) como en el cognitivo (19) y la necesidad de establecer una relación docente-estudiante de manera cálida (20), empática (21), reconfortante (22), de apoyo y desprovista de conflictos (20) no punitiva (20), con sentido del humor (23), que proteja la autoestima de los estudiantes (24), que favorezca su autonomía (25), que los apoye en la regulación de sus emociones (26) y que asegure a los estudiantes un ambiente propicio para su bienestar y desarrollo personal.

Por lo que corresponde a la relación didáctica, los trabajos de investigación que se interesan en la calidad de la relación docente-estudiante se apoyan en la noción de contrato didáctico, es decir, el conjunto de comportamientos del docente que son esperados por el estudiante y el conjunto de comportamientos del estudiante que son esperados por el docente (27), presentándose desde el preescolar y primero de primaria (28). Por ejemplo, Schubauer ha tratado el rol de las interacciones sociales en el desarrollo de las estructuras operatorias y ha analizado la relación docenteestudiante, y cómo estos últimos analizan los conocimientos bajo el efecto paralelo del plan cognitivo y socio afectivo y de las situaciones de enseñanza aprendizaje (29).

En cuanto al aprendizaje de la lectura y la escritura varios autores han señalado la importancia de recurrir en el preescolar a prácticas que favorezcan el desarrollo de la conciencia fonológica, las habilidades de decodificación, de fluidez, las estrategias de comprensión, de vocabulario, de escritura y las habilidades en ortografía (30); ya que las actividades de entrada a la escritura son integradas al programa de preescolar con el objetivo de preparar mejor a los niños para su ingreso en el primer año de primaria. De esta forma, si la relación docente-estudiante tiende a favorecer los aprendizajes y el desarrollo cognitivo de los niños, hay que tener siempre en cuenta que la afectividad y la cognición son indisociables y que el pensamiento se apoya, esencialmente, sobre las emociones y la volición (31).

Por tanto, el estudio de esta relación debe tener en cuenta los diferentes aspectos de la educación. Esto es lo que Pianta, La Paro y Hamre opinan considerando la relación docenteestudiante en su totalidad y teniendo en cuenta aspectos tanto cognitivos y organizacionales como socio afectivos (32). Igualmente, se ha constatado que a menudo es difícil distinguir los diferentes aspectos de esta relación.

Los métodos de investigación de la calidad de la relación docente-estudiante consisten, muy a menudo, en observar o describir el comportamiento tal como el Classroom Assessment Scoring System (CLASS) (32), instrumento 
que da acceso a la manera en cómo los docentes interactúan con sus estudiantes, reconociendo tres dominios: emocional, organizacional y cognitivo; con sus respectivas dimensiones como: el ambiente positivo (AP), la Sensibilidad de la Docente (SP), el respeto al punto de vista de los estudiantes (RPV), el manejo y regulación de los comportamientos (MRC), la productividad (P), el Apoyo al aprendizaje (AP), el desarrollo conceptual (DC), la calidad de las retroalimentaciones $(C R)$, y el modelar sobre el plan del lenguaje (MPL) (32) (Tabla 1).

\begin{tabular}{|c|c|c|}
\hline $\begin{array}{l}\text { Dominio (apoyo emocional, } \\
\text { organizacional y cognitivo }\end{array}$ & Dimension /sigla & Aspecto que valora \\
\hline \multirow{4}{*}{ Dominio emocional } & Ambiente positivo (AP) & $\begin{array}{c}\text { Relación positiva. } \\
\text { Afecto positivo. } \\
\text { Comunicación positiva. } \\
\text { Respeto hacia los estudiantes. }\end{array}$ \\
\hline & $\begin{array}{l}\text { Sensibilidad de la docente } \\
\text { (SP) }\end{array}$ & $\begin{array}{l}\text { Conciencia de las dificultades } \\
\text { experimentadas por el estudiante. } \\
\text { Apoyo a los estudiantes. } \\
\text { Solución de problemas. } \\
\text { Confort de los estudiantes. }\end{array}$ \\
\hline & $\begin{array}{l}\text { El respeto al punto de vista } \\
\text { de los estudiantes (RPV) }\end{array}$ & $\begin{array}{l}\text { Flexibilidad y énfasis educativo. } \\
\text { Autonomía y liderazgo de los estudiantes } \\
\text { Expresión de los estudiantes. } \\
\text { Libertad de movimientos. }\end{array}$ \\
\hline & $\begin{array}{l}\text { El manejo y regulación de los } \\
\text { comportamientos (MRC) }\end{array}$ & $\begin{array}{l}\text { Expectativas claras en cuento a los } \\
\text { comportamientos esperados. } \\
\text { Actitud proactiva. } \\
\text { Reorientación de los comportamientos } \\
\text { inadecuados. } \\
\text { Comportamientos de los estudiantes. }\end{array}$ \\
\hline \multirow{2}{*}{$\begin{array}{l}\text { Dominio } \\
\text { Organizacional }\end{array}$} & La productividad $(\mathrm{P})$ & $\begin{array}{c}\text { Aprovechamiento del tiempo de manera } \\
\text { eficaz. } \\
\text { Aprendizaje continuo. }\end{array}$ \\
\hline & Apoyo al aprendizaje (AP), & $\begin{array}{l}\text { Enfoque en materiales didácticos. } \\
\text { Mantener el interés del estudiante. }\end{array}$ \\
\hline \multirow{3}{*}{ Dominio Cognitivo } & El desarrollo conceptual (DC) & $\begin{array}{c}\text { Análisis y razonamiento. } \\
\text { Creatividad. } \\
\text { Integración de conocimientos. } \\
\text { Relación con la realidad de los estudiantes. }\end{array}$ \\
\hline & $\begin{array}{c}\text { Calidad de las } \\
\text { retroalimentaciones (CR) }\end{array}$ & $\begin{array}{c}\text { Andamiaje. } \\
\text { Intercambios sostenidos. } \\
\text { Reflexiones sobre los procesos. } \\
\text { Precisión de la información. } \\
\text { Estimular al estudiante. }\end{array}$ \\
\hline & $\begin{array}{l}\text { Modelar sobre el plan del } \\
\quad \text { lenguaje (MPL) }\end{array}$ & $\begin{array}{l}\text { Conversaciones. } \\
\text { Preguntas abiertas. } \\
\text { Repetición y elaboración. } \\
\text { Acompañamiento verbal. } \\
\text { Calidad de la lengua. }\end{array}$ \\
\hline
\end{tabular}

Fuente: Elaboración por los autores basado en Pianta RC, La Paro K, Hamre B. Classroom assessment scoring system (CLASS) (32). 
Bridget, Hamre en unión con Pianta (33) realizan un estudio sobre la relación docente-estudiantes examinando las maneras como el riesgo de los niños de fracaso escolar puede ser moderado por el apoyo de profesores. Estudiaron 910 niños ente 5 y 6 años de edad, en un estudio nacional prospectivo. Al final del primer grado, los estudiantes en riesgo colocados en las aulas de primer grado, que ofrecían un fuerte apoyo instruccional y emocional, tuvieron mejores resultados escolares, a diferencia de los estudiantes en riesgo en las aulas menos favorables que tuvieron menor rendimiento y más conflicto con los maestros.

Por otra parte, Venet y Schmidt (17) investigaron las condiciones favorables al desarrollo académico y el logro de estudiantes con dificultades de aprendizaje en las clases regulares de quinto y sexto grado. Se enfocan en las opiniones subjetivas de directores sobre sus estrategias de inclusión educativa. Los resultados muestran que la evolución de una escuela es un largo proceso de coconstrucción de significados compartidos entre los individuos de esa comunidad, el estudio plantea que estos directores son guiados por la adaptabilidad a su entorno y actúan de acuerdo con principios basados en una relación afectiva positiva que coinciden con sus miembros del personal. Estos hallazgos proporcionan una visión de las maneras en que los directores integran sus enfoques y creencias acerca de incluir a los estudiantes con dificultades en su trabajo general como líderes para obtener mejor rendimiento escolar de sus estudiantes, en conjunto con una relación afectiva positiva.

Una visión que complementa los anteriores estudios es la de Betancourth, Burbano y Venet quienes realizan una investigación que buscaba conocer la coherencia entre el discurso y el actuar de una docente con respecto a las relaciones docente-estudiantes en preescolar. Fue un estudio cualitativo de estudio de caso único. Se trabajó con una docente y sus 35 estudiantes de preescolar, los niños tenían entre 4 y 5 años de edad. Se observaron tres clases a lo largo del periodo escolar: inicio, mitad y final de año, con el objetivo de observar los cambios de la docente en sus relaciones con los niños. Se filmaron 20 minutos por cada clase. Adicionalmente, se realizó una entrevista semiestructurada con la docente, a medio año, para comparar con las clases observadas. Los datos fueron analizados por medio de los dominios, dimensiones y características del instrumento CLASS de Pianta. La investigación evidenció incoherencias entre el discurso y el actuar de la docente con referencia a los ámbitos afectivo y cognitivo; igualmente, con los lazos relacionales en la formación en el ambiente del aula de clases. Es decir, la docente tiene claro que el afecto es un factor importante en la formación del estudiante, sin embargo, no logra actuar en sus clases acorde a esta concepción(34).

De esta manera, se propuso realizar una investigación que permitiera determinar la relación socioafectiva de docenteestudiantes de preescolar y primero de primaria a través de los comportamientos del docente utilizando el Class de Pianta para su evaluación.

\section{Metodología}

Estudio cuantitativo descriptivo exploratorio, se desarrolló en dos instituciones educativas públicas de la ciudad de Pasto, Nariño, Colombia. La muestra fue intencional conformada por 14 docentes, de sexo femenino, de las cuales 9 docentes eran de preescolar y 5 de primaria, quienes aceptaron participar del estudio de manera voluntaria y que tenían a su cargo 436 niños y niñas en los grados de preescolar y primaria.

Se solicitó permiso a las instituciones educativas y cada una de las docentes firmó el consentimiento informado correspondiente. Es de aclarar que los datos de la investigación son de los comportamientos de los docentes a partir de la observación de sus clases las cuales fueron grabadas previa autorización de los docentes en tres momentos durante el transcurso del año escolar.

El instrumento de recolección y análisis de información fue el CLASS (Classroom Assessment Scoring System) entendido como una pauta de observación que se compone de tres dominios: apoyo emocional, apoyo organizacional y apoyo cognitivo, cada uno se subdivide en dimensiones (generalmente cuatro) que a su vez se subdividen en características, que se componen de conductas que se pueden observar (Tabla 1) (32). De esta manera, el CLASS describe los comportamientos esperados de los docentes que saben contribuir al desarrollo socio afectivo y cognitivo de sus estudiantes, estableciendo una relación positiva con ellos, es decir, una relación que les da un sentimiento de seguridad tanto en el plano afectivo como cognitivo, sentimiento que permite a los estudiantes explorar su entorno. Respecto al instrumento Del CLASS de Pianta no se encontró una estandarización en un país de habla hispana, sin embargo, aparece en acceso libre en línea para su uso y es innovadora su aplicación en Colombia, por lo tanto las investigaciones que han utilizado este instrumento son limitadas, pero han arrojado información valiosa, verificando que el CLASS es una herramienta para observar la efectividad de estas interacciones.

El procedimiento utilizado se inició con la recolección de datos a través de observaciones de clase de las docentes participantes en este estudio, grabadas en video; las filmaciones se realizaron tres veces en el año escolar, al inicio (marzo-abril), mitad (junio-agosto) y final del año (octubre-noviembre), durante 20 minutos de clase. La realización de la investigación fue conocida y autorizada por las directivas y docentes de las instituciones educativas, con quienes se organizó un calendario de observación. El equipo investigador realizó el análisis de los videos (previamente las investigadoras recibieron capacitación para hacer la respectiva medición con el instrumento), a 
partir de la grilla del CLASS para establecer un acuerdo inter jueces. La calificación va desde uno como puntaje más bajo y siete como el puntaje mayor en relación al cumplimiento de los comportamientos de los docentes determinados en cada dimensión del CLASS. Las calificaciones se agrupan en los rangos 1 y $2=$ bajo; 3,4 y $5=$ medio; 6 y $7=$ alto (32).

A partir de las calificaciones dadas de cada video, por las investigadoras como interjueces, se analizan los resultados estadísticamente teniendo en cuenta la relación de variables intra-sujeto, es decir, las diferencias significativas entre cada una de las dimensiones a través del tiempo, teniendo en cuenta las tres observaciones realizadas en el año escolar y la relación de variables inter-sujeto que son las diferencias significativas del promedio total de las dimensiones y las variables: institución, grado, número de estudiantes y docentes. Las dimensiones de la relación docenteestudiante planteadas por Pianta se analizaron a través del modelo lineal general desde las medidas repetidas. Para esto se analizó el criterio de esfericidad de Mauchly, y el análisis de la relación variables sociodemográficas con el promedio total de la evaluación se analizó a través del estadístico Mann Whitney considerando específicamente la significancia bilateral (P) en donde la calificación menor a 0.05 demuestra que hay diferencias significativas.

\section{Resultados}

Respecto a las características de los participantes se trabajó con 14 docentes, 9 de preescolar y 5 de primero de primaria, que hacían parte de dos instituciones educativas de la ciudad de Pasto, todas las docentes de sexo femenino. Es así que, de la relación intra-sujeto (dimensión-tiempo), se observa que en ocho de las nueve dimensiones no existen diferencias significativas a lo largo del tiempo, por lo tanto, las docentes mantienen la misma forma de relacionarse durante todo el año escolar en los aspectos afectivo y de aprendizaje (Tabla 2).

Sin embargo, en la dimensión MPL los resultados fueron distintos, ya que se presenta una diferencia significativa durante el tiempo en las diferentes observaciones del año escolar.

Se observa que solamente en la dimensión CR la prueba de esfericidad de Mauchly se encuentra en 0.033 , lo que significa que no cumple con el criterio de esfericidad, por esta razón solo en la dimensión CR se tendrá en cuenta la medición $\mathrm{F}$ en la esfericidad asumida; que corresponde a 0.527 (Tabla 3), lo cual permite que se cumpla con el criterio de esfericidad.

En cuanto a la relación de las variables institución, grado, número de estudiantes y docentes con el promedio total de la evaluación que se analizó a través del estadístico intersujeto con las medidas no paramétricas de Mann Whitney, como se dijo anteriormente, se encontró que las variables institución, grado y docentes no tienen diferencia significativa entre sí, manifestando que las docentes de diferentes instituciones mantienen un comportamiento similar a lo largo del año, tanto en el grado de preescolar como primero. La variable número de estudiantes se

Tabla 2. Relación intra-sujeto (dimensión-tiempo)

\begin{tabular}{|c|c|c|c|c|c|c|c|c|c|c|}
\hline & Dimensiones & AP & SP & RPV & MRC & $\mathbf{P}$ & AP & DC & CR & MPL \\
\hline \multirow{3}{*}{ 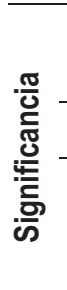 } & $\begin{array}{l}\text { Esfericidad } \\
\text { asumida }\end{array}$ & 0.157 & 0.839 & 0.314 & 0.623 & 0.947 & 0.829 & 0.109 & 0.60 & 0.016 \\
\hline & Límite inferior & 0.182 & 0.681 & 0.291 & 0.500 & 0.818 & 0.671 & 0.144 & 0.48 & 0.046 \\
\hline & $\begin{array}{l}\text { Prueba de } \\
\text { esfericidad de } \\
\text { Mauchly }\end{array}$ & 0.556 & 0.561 & 0.745 & 0.746 & 0.444 & 0.919 & 0.991 & 0.033 & 0.404 \\
\hline
\end{tabular}

*AP: Ambiente positivo; SP: Sensibilidad del Docente. RPV: Respeto al punto de vista de los estudiantes. MRC: Manejo y regulación de los comportamientos. P: Productividad. AP: Apoyo al aprendizaje. DC: Desarrollo conceptual. CR: Calidad de las Retroalimentaciones. MPL: Modelar sobre el plan del lenguaje.

Tabla 3. Esfericidad asumida. Dimensión calidad de las retroalimentaciones

\begin{tabular}{lccccc}
\hline & $\begin{array}{c}\text { Tipo III de suma } \\
\text { de cuadrados }\end{array}$ & gl & $\begin{array}{c}\text { Cuadrático } \\
\text { promedio }\end{array}$ & F & Sig. \\
\hline Esfericidad asumida & 0.571 & 2 & 0.286 & 0.527 & 0.597 \\
\hline
\end{tabular}


observa una diferencia significativa con respecto al promedio total (Tabla 4).

Finalmente, en la Tabla 5 se presenta el promedio total de la aplicación del CLASS. Once de las 14 docentes evaluadas se encuentran en un rango 2, es decir, una calificación media que va entre las puntuaciones 3,4 y 5 según el CLASS de Pianta, lo que significa que la relación de las docentes con los estudiantes durante todo el año escolar se mantuvo, sin cambios significativos.

\section{Discusión}

En el documento de la UNESCO (35) se hace énfasis en la relación entre docente-estudiantes la cual debe mejorar durante el transcurso del año escolar, y es evidente en la frase de una docente de primaria "ahora entiendo a la escuela como un sitio donde vamos a aprender y mejorar, donde compartimos el tiempo, el espacio y el afecto con los demás" (36). Estos son tres aspectos importantes que se deben compartir especialmente con niños de preescolar, porque para ellos el ingreso a la escuela constituye la primera separación de la madre durante varias horas del día (37), por esta razón es imperioso que las docentes de preescolar reconozcan el afecto como un elemento importante en el crecimiento de los niños, y esto se puede lograr a través de la profunda inclusión del tema en diversas materias durante la educación profesional en la universidad o con capacitaciones durante el tiempo de trabajo. Se realiza énfasis en esto, porque se observa en los resultados que el método de trabajo se ha tornado monótono en la mayoría de las docentes. Por eso, es necesario que los docentes reconozcan que las nuevas demandas inducen un cambio de perspectiva, nuevos estilos de enseñanza, en un marco de permanente revisión y cuestionamiento (36).

El lenguaje fue la única dimensión con diferencias significativas durante el año escolar. Podría adjudicarse esa distinción, por ser un elemento que está en continuo desarrollo. Sin embargo, este aspecto se debe aprovechar aún más en esta etapa del desarrollo del niño, ya que es el elemento que permite que la relación mejore, además es lo esencial del enfoque de Vigotsky, el cual consiste en considerar al individuo como el resultado del proceso histórico y social donde el lenguaje desempeña un papel esencial. Desarrollo que se puede relacionar con diferentes estudios donde mencionan que el cambio en el lenguaje sí se observa directamente proporcional a la edad de los niños, y específicamente en la etapa de preescolar y primaria (37).

Por otro lado, las docentes de preescolar y primero de primaria de diferentes instituciones mantienen similar desarrollo de la enseñanza-aprendizaje en el aula de clase, esto podría suceder porque responden a leyes del Ministerio de Educación, que les exige desarrollar determinados temas para los niños y niñas preescolares, lo cual es adecuado para mantener un orden en los pasos de la enseñanza, y que también se da en diferentes países, ya que cada docente se rige a la organización correspondiente. Sin embargo, es relevante que las docentes comprendan que ellas tienen la capacidad y las habilidades de crear e imaginar nuevas maneras de enseñar la temática establecida, teniendo siempre presente que la metodología de enseñanza responde a una herramienta concreta para transmitir los contenidos, procedimientos y principios al estudiante, y cumpliéndose con los objetivos de aprendizaje determinados (38). En este sentido, se afirma que el docente debe estar en constante formación para enfrentar los retos que implica día a día la enseñanza (39).

Al tener cursos conformados por una cantidad significativa de estudiantes podría generar en las docentes inconvenientes en estar atentas a todos los niños y niñas, y por ende el aumento de una adecuada relación entre estos dos actores podría verse afectada. Por esto, es preciso considerar un espacio físico bastante amplio que se conforme de una cantidad apropiada de aulas (40).

Tabla 4. Relación inter-sujeto

\begin{tabular}{ccccc}
\hline \multicolumn{4}{c}{ Total de las variables relacionado con } \\
\hline $\begin{array}{c}\text { Variable } \\
\text { independiente }\end{array}$ & Institución & Grado & $\begin{array}{c}\text { Número de } \\
\text { estudiantes }\end{array}$ & Docentes \\
\hline $\begin{array}{c}\text { Significancia } \\
\text { bilateral }(\mathrm{P})\end{array}$ & 0.530 & 0.925 & 0.040 & 1.000 \\
\hline
\end{tabular}

Tabla 5. Promedio total de la aplicación del CLASS de Pianta

\begin{tabular}{ccc}
\hline Rango & Frecuencia Docentes & Porcentaje \\
\hline 2 & 11 & 78.6 \\
\hline 3 & 3 & 21.4 \\
\hline Total & 14 & 100.0 \\
\hline
\end{tabular}


Adicionalmente, se considera para mejorar la relación entre docente y estudiantes de preescolar, es a través de la variación en la dinámica de clase en el día a día, de la continua escucha a los estudiantes y el análisis de los comportamientos y actitudes de cada niño desde el principio del año (41).

Para lograr el progreso positivo en la relación es necesario involucrar el componente del afecto y la comunicación para conseguir la comprensión mutua, la percepción interpersonal y la empatía (15); teniendo siempre presente que se aborda el afecto desde una relación indisociable con la cognición como se afirma en las obras de Vygosky.

Finalmente, los nuevos conocimientos extraídos del estudio contribuyen a nutrir el abordaje socio constructivista precisando la naturaleza del vínculo entre afecto y cognición para la clarificación de la relación docenteestudiante, relación que se percibe como fundamental para favorecer la comprensión de las invariables del desarrollo humano. Además, esto lleva a mejorar los métodos de enseñanza desde una perspectiva docente a partir de la comprensión de las relaciones complejas y sutiles que se tejen entre el afecto y el intelecto.

\section{Conclusiones}

En conclusión, se puede observar que ocho de las nueve dimensiones del instrumento CLASS se mantienen igual durante la mayor parte del tiempo del año escolar. Adicionalmente, se menciona que para mejorar la relación docente-estudiantes es necesario que exista un programa de intervención con el objetivo de trasmitir el conocimiento sobre el CLASS de Pianta para que así las docentes tengan un esbozo de todas las herramientas que compone, lo que se podría mencionar como, una buena y positiva relación entre los docentes y estudiantes, no solo de preescolar y primero de primaria sino también de los siguientes grados escolares.

Finalmente, es importante mencionar que para futuras investigaciones es necesario realizar más de tres grabaciones durante el año escolar, además de hacer una observación no solo dentro del aula de clase sino también en los tiempos de receso.

\section{Responsabilidades éticas}

Protección de personas y animales. Los autores declaran que en esta investigación no se han realizado experimentos en seres humanos ni en animales.

Confidencialidad de los datos. Los autores declaran que han seguido los protocolos de su centro de trabajo sobre la publicación de datos de pacientes y que todos los pacientes incluidos en el estudio han recibido información suficiente y han dado su consentimiento informado por escrito para participación en dicho estudio.

Derecho a la privacidad y consentimiento informado. Los autores declaran que han obtenido el consentimiento informado de los pacientes y/o sujetos referidos en el artículo. Este documento obra en poder del autor de correspondencia.

\section{Conflicto de intereses}

Las autoras declaran no tener conflicto de interés.

\section{Financiación}

La presente investigación fue financiada por el Sistema de Investigaciones de la Universidad de Nariño, Colombia.

\section{Referencias}

1. González E. Educar en la afectividad. [Internet]. 2010. Universidad Complutense. Madrid. [Citado 2017 noviembre 09]. Disponible en: https://educrea.cl/educarla-afectividad/

2. Avalos B. La formación docente inicial en Chile. [Internet]. 2010. [Citado 2017 noviembre 09]. Disponible en: https://www.researchgate.net/profile/Beatrice Avalos/pu blication/266338903 LA FORMACION DOCCENTE I NICIAL_EN_CHILE/links/5548d15a0cf2 $\overline{7} \mathrm{c} 5000677 f 5 \overline{\mathrm{e}}$. pdf.

3. Ministerio de Educación Quebec-MEQ. Programme de formation de l'école québécoise, Éducation préscolaire et enseignement primaire, Version approuvée. [Internet]. 2011 [citado 2016 diciembre 30]. Disponible en: http://www.education.gouv.qc.ca/fileadmin/site_web/ documents/dpse/formation_jeunes/prform2001.pdf.

4. Vygotsky L. Obras completas. Tomo 5. 1997. Madrid España: Visor Dis.

5. Rogoff BS. Cognition as a collaborative process. Handbook of Child Psychology. [Internet]. 2011. [Citado 2017 noviembre 16]. Disponible en: http://psy cnet.apa.org/record/2005-01927-013.

6. Páez D, Carbonero A. Afectividad, cognición y conducta social. Psicothema. 1993; 5(1):133-150.

7. Piaget J. Inteligencia y afectividad. [Internet]. 2011. [Citado 2017 febrero 12]; Disponible en: http://www. verticespsicologos.com/sites/default/files/Inteligenciay-afectividad.

8. Gil N, Blanco L, Guerrero E. El dominio de afectivo en el aprendizaje de las matemáticas. Una revisión de sus descriptores básicos. Revista iberoamericana de educación matemática. 2005; 2:15-32.

9. Vygotsky L. Pensamiento y lenguaje. 1978. 2 ed. Argentina: La Pleyade. 494 p.

10. Vygotsky L. Vygotsky's educational theory cultura context. Reino Unido. International center for the enhancement of learning potential. [Internet]. 2011. Disponible en: http://www.icelp.info/. 
11. Bowlby J. Reasonable fear and natural fear. Int J Psychiatry. 1970-1971; 9:79-88.

12. Atkinson C, Regan T, Williams C. Working collaboratively with teachers to promote effective learning. Support for learning. 2006; 21(1):33-39. Disponible en: http://doi.org/10.1111/j.1467-9604.2006.00398.x

13. Baker S. Sustainable Development. 2006. 3 ed. Estados Unidos: Routledge.

14. Roorda D, Koomen H, Spilt J, Oort F. The influence of affective teacher-student relationships on school engagement and achievement: A meta-analytic approach. Review of Educational Research. 2011; 81(4):493-529. Disponible en: https://doi.org/ 10.3102/0034654311421793

15. Escobar F. Importancia de la educación inicial a partir de la mediación de los procesos cognitivos para el desarrollo humano integral. Revista de educación Laurus. 2006; 21(12):169-194.

16. Hamre B, Pianta R. Can Instructional and Emotional Support in the First-Grade Classroom Make a Difference for Children at Risk of School Failure?. Child Development. 2005; 76(5):949-967. Disponible en: https://doi.org/10.1111/j.1467-8624.2005.00889.x.

17. Venet M, Schmidt S. Principals Facing Inclusive Schooling or Integration. Canadian journal of education. 2012; 35(1):217-38.

18. Teven J, McCroskey J. The relationship of perceived teacher caring with student learning and teacher evaluation. Communication Education. 1997; 46(1):117. Disponible en: https://doi.org/10.1080/ 03634529709379069

19. Venet M, Schmidt S, Paradis A, Ducreux E. La qualité de la relation entre l'enseignante et ses élèves: une simple affaire de cœur? In S. Schmidt (dir.), les conditions favorables au cheminement et à la réussite scolaires d'élèves en difficulté d'apprentissage en classe ordinaire au troisième cycle du primaire: la pratique d'enseignement de Calypso. 3 ed. Canada. PUQ; 2009.

20. Pianta R. Student-teacher, relationship Scale (STRS). Professional Manual. [Internet]. 2001. [citado 2017 noviembre 19]. Disponible en: http://schd.ws/ hosted_files/ulead2016/3d/Studnet\%20Teacher\%20Rel ationship\%20Scale\%20(short\%20form).

21. Fijalkow J, Nault T. La gestión de la clase. [Internet]. 2010. [citado 2017 enero 24]. Disponible en: https://rua.ua.es/dspace/bitstream/10045/64471/1/Psic ologia-y-educacion_141.pdf.

22. Young $\mathrm{C}$, Koopsen $\overline{\mathrm{C}}$. Health and healing. [Internet]. San Diego: 2011. [citado 2017 febrero 15]. Disponible en: https://journals.equinoxpub.com/index.php/HSCC/articl e/view/16929.

23. Gardner H. Multiple intelligences: New horizons. [internet] Nueva York: 2006. [citado 2017 enero 13]. Disponible en: https://howardgardner.com/m-i-newhorizons/.

24. Frymier A, Houser M. The teacher - student relationship as an interpersonal relationship. Communication Education. 2000; 49(3):207-219. Disponible en: https://doi.org/10.1080/03634520009379209

25. DeVries R. Coercion in constructivist education. Paper presented at the annual meeting of the American Educational Research Association. [Internet]. 2001. [citado 2017 enero 16]. Disponible en: https://pdfs.semanticscholar.org/3194/a7b93239bd633 05323b0576fee58f350d0aa.pdf.

26 . Anh J. A good teacher in every classroom: Preparing the highly qualified teachers our children deserve. 2005. 2 ed. San Francisco: DarlingHammond.

27. Brousseau G. Théorie des Situations Didactiques. Grenoble. Psychologie et problèmes de la société. [Internet]. 2013. [citado 2017 enero 13]. Disponible en: http://math.univ-Iyon 1.fr/capes/IMG/pdf/UE10CoursSSLno1.pdf

28. Fernandez, P, Extremera N. La Inteligencia Emocional y la educación de las emociones desde el Modelo de Mayer y Salovey. Revista Interuniversitaria de Formación del Profesorado. 2005; 19(3):63-93.

29. Schubauer M. El desarrollo cognitivo de los niños en la escuela primaria: la psicología del aprendizaje en las diferentes situaciones pedagógicas. Revista de educación. 1986; 279:103-120.

31. Vygotsky L. Obras escogidas II. 1993. Madrid - España. Visor.

32. Pianta R, La Paro K, Hamre B. Classroom assessment scoring system (CLASS). [Internet]. 2008. [citado 2017 enero 07]. Disponible en: http://curry.virginia.edu/ research/centers/castl/class.

33. Monje C. Metodología de investigación cuantitativa y cualitativa. Guía práctica. [Internet]. 2011. [citado 2017 febrero 13]. Disponible en: https:// carmonje.wikispaces.com/file/view/Monje+Carlos+Artur o + - + G u \% C 3 \% A D a + di d \% C 3\% A 1 c t i c a + Metodolog $\%$ C3\%ADa+de+la+investigaci\%C3\%B3n.

34. Betancourth S, Burbano D, Venet M. La relación docenteestudiantes de preescolar según el CLASS de Pianta. Psicogente. 2017;20(37):55-69.

35. UNESCO. Protagonismo docente en el cambio educativo: PRELAC. 2005: p. 193. Costa Rica.

36. Sebastiani I. El comportamiento del niño en la escuela Revista de educación, cultura y sociedad. [Internet] 2013. [citado 2017 enero 13]. Disponible en: https://issuu.com/gorettijimenez/docs/reporte_de_inves tigaci_n

37. Hernández M. Metodologías de enseñanza y aprendizaje en altas capacidades. 2010. 3 ed. Santa Cruz de Tenerife.

38. Pérez A. Aprender a educar. Nuevos desafíos para la formación de docentes. Revista Interuniversitaria de Formación del Profesorado. 2010; 68(24,2):37-60.

39. Barrera M. El docente como gestor del clima en el aula. (Tesis de maestría). Cantabria, España. 2012.

40. Gómez C, Sanz A, Puyal E, Luna M, Sanagustín M, Elboj C. Convivencia en los centros educativos. 2011 Convivencia docente y alumnos. Zaragoza. GORFISA.

41. Treviño E, Varela C, Romo F, Núñez V. Presencia de lenguaje académico en las educadoras de párvulos y su relación con el desarrollo del lenguaje de los niños. Calidad de la educación. 2015; 43:137-168. Disponible en: ht tp://dx.doi.org/10.4067/S 0718 45652015000200005 . 